\title{
Spiral Galaxy in 3D as Seen with SpIOMM
}

\section{Laurie Rousseau-Nepton, Carmelle Robert and Laurent Drissen}

Université Laval \& Centre de Recherche en Astrophysique du Québec, Québec, Canada

\begin{abstract}
Using the imaging Fourier transform spectrograph (FTS) SpIOMM we study 7 nearby spiral galaxies. The large database of spectra obtained around $\mathrm{H} \alpha$ and $\mathrm{H} \beta$ is ideal to study the star forming regions and warm ionized medium (WIM) with a high spatial resolution $(\sim 50-150 \mathrm{pc})$.
\end{abstract}

Keywords. galaxies: nearby spiral, galaxies: evolution - galaxies: star formation

\section{SpIOMM}

SpIOMM, the imaging FTS of the Mont-Mégantic Observatory, offers a large FOV(12'x12'), a good spatial resolution of $1.1 "$ (435 500 pixels and spectra), and a spectral coverage in selected bandpasses of the visible with an adjustable resolution from 1 to 20 000. Two data cubes have been obtained ( $\sim 5 \mathrm{hrs} /$ cube) for each galaxy covering the spectral domains of 4750-5150 $\mathrm{A}(\mathrm{R} \sim 650)$ and 6500-6800 A $(\mathrm{R} \sim 2000)$.

\section{HII Regions and the WIM}

Hil regions (HIIR) are detected over the whole galactic disks. For example, we identified 566 HiIR with a SNR > 8 on NGC628. Also, emission from a WIM (Haffner \& al. 1999; Blanc \& al. 2009) can be detected beyond the HiIR boundaries (Fig. 1). The WIM can be related to the HIIR escaping photons, but also to $\mathrm{SNe}, \mathrm{PNe}$, and other sources including AGB stars, AGN, and shocks. These ionizing sources are not necessary physically linked together and produce very different physical conditions in the surrounding gas. For NGC 628, the metallicity gradient calculated after taking into account the effect of the WIM becomes steeper (Fig. 2; Rousseau-Nepton et al. in prep).

\section{References}

Blanc, G. A., et al. 2009, ApJ, 704, 842

Haffner, L. M., et al. 1999, ApJ, 523, 223

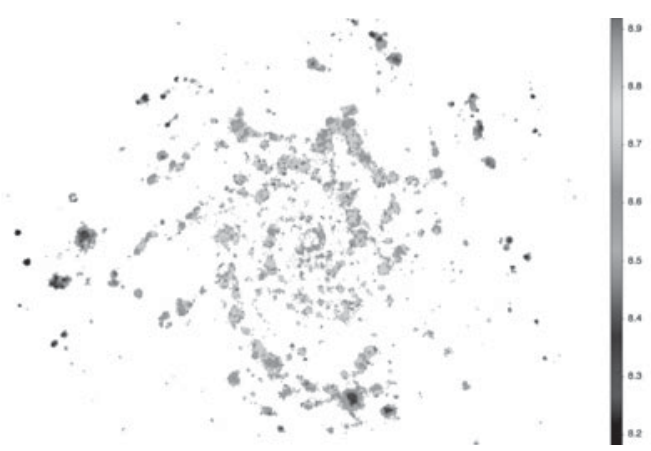

Figure 1. NGC 628 metallicity map using the O3N2 indicator for all spaxels above $4 \sigma$.

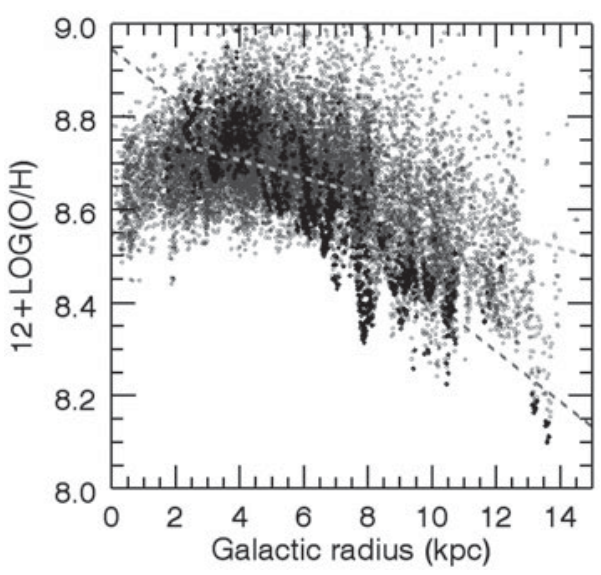

Figure 2. NGC 628 metallicity gradient. Blue dots and the orange dashed line are measurement in all spaxels above $4 \sigma$. Black dots and the red dashed line are from selected pixels in HiI regions. 